\title{
Analysis of Correlation between Interleukin-6 Gastric Mucosa with Plasma Cortisol Level in Functional Dyspepsia with Psychological Stress
}

\author{
Arina Widya Murni ${ }^{1}$, Nita Afriani ${ }^{2}$, Saptino Miro ${ }^{3}$ \\ \{arinawidyamurni@med.unand.ac.id ${ }^{1}$, nitaafriani83@med.unand.ac.id ${ }^{2}$, saptinomiro@med.unand.ac.id ${ }^{3}$ \} \\ Department of Internal Medicine Subdivision Gastroenterohepatology Medicine, Faculty of \\ Medicine, Universitas Andalas
}

\begin{abstract}
Pathophysiology of Functional dyspepsia still unclear, many factors had been a contribution. Cortisol was hormone stress that releases from cortex adrenal through activation of the Hypothalamus-Pituitary-Adrenal Axis (HPA axis). It could affect immunity in the gastrointestinal mucosa and affected of IL-6 expression. This study aimed to determine the correlation of expression IL-6 in gastric mucosa with cortisol level in patients with functional dyspepsia with stress. It was a cross-sectional study with consecutive sampling method. There are 80 patient got a blood sampling, endoscopic and mucosal biopsy - screening stress with DASS 42. Morning and evening Cortisol plasma was examined with Elysa, immunohistochemical methods examined IL-6. 80 patients meet the criteria, divided in to stress group and non-stress group with the range of age 18-65 years. The mean value of morning cortisol was $15,79 \pm 7,6 . \mu \mathrm{g} / \mathrm{dL}$ and evening cortisol was $6,43 \pm 4,53 \mu \mathrm{g} / \mathrm{dL}$ at group with no stress. In group with stress the cortisol morning was $24,03 \pm 12,18 \mu \mathrm{g} / \mathrm{dL}$ and cortisol evening was $12,42 \pm 9,25 \mu \mathrm{g} / \mathrm{dL}$. Mean value of expression of IL-6 In gastric mucosa was $72,95 \pm 19,49$ in the stress group and $73,28 \pm 16,70$ in the non-stress group There was a significant correlation between morning plasma cortisol with expression IL-6 in the stress group $(\mathrm{p}=0,05)$ and no significant result in the non-stress group.
\end{abstract}

Keywords: Cortisol plasma, Expression IL-6, Psychological Stress.

\section{Introduction}

Prevalence of dyspepsia in the world is $5 \%-40 \%$ of the population. About $60 \%$ of these is functional dyspepsia (FD). Mahadeva and Goh's study (2006) states that FD patients roughly $20 \%$ go to general practitioners and specialist, $50 \%$ get long-term treatment and $30 \%$ of them have to leave work or school due to illness.[1]

Many factors are thought to contribute to the pathophysiology of functional dyspepsia. The psychosocial stress factors, genetic, $H$ pylori infection and other microbiota, altered gastrointestinal tract infections, abnormalities of gastric motility and visceral hypersensitivity problems have a contribution to be causes of FD. Psychological factors associated with sympathetic hyperactivity and elevated plasma cortisol levels due to hyperactivity of the corticotropin hormone (CRH).[2][3]

Gastrointestinal functional impairment due to psychological problems occurs through activation of the Hypothalamus-Pituitary-Adrenal Axis (HPA axis). Affected pathways are 
neurogenic and hormonal, lead to increasing the cortisol hormone that known as the stress hormone. Hormonal pathways can be explained by proven hypothalamic activation during the occurrence of emotional stress exposure that causes the release of corticotrophin releasing hormone (CRH) from the anterior pituitary. Furthermore, CRH will activate the adrenal cortex releasing adrenocorticotropin releasing hormone, and produce cortisol which will affect much of the various systems in the body.[4]

Cortisol hormone will stimulate the production of stomach acid (aggressive factor) and can inhibit prostaglandins (defensive factors) that are protective in the stomach. Reduced prostaglandins for the next will facilitate the occurrence of damage to the gastric mucosa. Andreas's (2005) study proved that there was a significant increase in the value of cortisol in FD compared to the control group. Similarly, Murni study (2006) found a significant increase in morning cortisol in FD patients with depressed $(p=0.019)$. [5] [6]

Cortisol is a hormone produced by the fasciculata zone and the reticular zone in the adrenal cortex. The production of cortisol is triggered by the stimulation of the hormone ACTH secreted by the pituitary gland. The pituitary releases ACTH by activation of CRH which is the main regulator of cortisol secretion. It takes a short time for $\mathrm{CRH}$ secretion, peak before morning time (before $8 \mathrm{am}$ ) and in the afternoon (before $8 \mathrm{pm}$ ) is known as the diurnal phase. There is a feedback mechanism in the regulation of the amount of cortisol in the blood, wherein the cortisol released in the circulation may give negative feedback to the hypothalamus in releasing the $\mathrm{CRH}$ .[7]

Some inflammatory markers were found to increase in patients with FD, such as TNF a, IL 6, il-12, IFN gamma, IL-1B, IEL / CD3 / CD 25, TLR4, anti-flagellin antibody and there was eosinophil infiltration in the duodenum and elevated esosinophils in fluid Stomach. Plasmasoluble Interleukin-6 is a loose form after binding to IL-6R receptors when circulating in the blood can stimulate the adrenal hypothalamus pituitary (HPA) axis. Stress will affect IL-6 through these HPA axis pathways Studies have shown that individuals who can reduce their stress levels will show improvement in immunity. [8] [9]

Psychological stress as a trigger in increasing the hormone cortisol in plasma will affect immune system dysfunction in the gastric mucosal defence and can trigger the activity of $\mathrm{H}$. Pylori. Patients with functional dyspepsia, although no mucosal or hyperemic abnormalities are present, are likely to progress to organic diseases such as ulcers and cancers secretly, so management of functional dyspepsia about psychological stress management is important, as it controls plasma cortisol levels, suppressing dysfunction immune system and reduce $\mathrm{H}$. pylori activity.[10]

\section{Methods}

It is a cross-sectional study conducted in Mei 2015 until March 2017 on dyspepsia syndrome patients from Andalas health centre and Padang Pasir health centre, as well as outpatients of M. Djamil General Hospital, Padang.

\subsection{Patients Selection}


Eighty patients who enrolled in this study have a range of age 18-65 years, suffered dyspepsia syndrome for more than two months. There are no signs of bleeding (alarm symptoms), no history of chronic diseases, not pregnant and are not under medication. All patients were tested for psychological stress using Depression Anxiety and Stress Scale (DASS 42). Esophagogastroduodenoscopy (EGD) was done to determine the underlying causes of the symptoms, and mucosal tissues ware taken during EGD. This study was approved by the Research Ethics Committee of Medical Faculty, Andalas University. (No 08/KEP/FK / 2015)

\subsection{Blood cortisol}

Serum samples were taken from patients and examined using Elecysys Cortisol Reagen Kit and Electrochemiluminescence Immunoassay system (ECLIA) on device Roche Elecsys 1010/2010 with modular analitycs $\mathrm{E} 170$ with normal adult values of

4.30-22.40 $\square \mathrm{g} / \mathrm{dL}$ in the morning serum and 3.09-16.66 $\square \mathrm{g} / \mathrm{dL}$ in evening serum.

$4.30-22.40 \mu \mathrm{g} / \mathrm{dL}$ Note: Adult, Age: $>18$ years (Morning serum)

$3.09-16.66 \mu \mathrm{g} / \mathrm{dL}$ Note: Adult, Age: $>18$ years (Evening serum)

\subsection{Histopathology Assessment with Immunohistochemical Methods}

Immunohistochemistry of gastric tissue is taken from two locations, antrum, and fundus, Once the tissue harvested, the fixation begins with a paraffin block. IHC staining process is done to examine the expression of IL-6.

Antigen detection in tissues and cells are a multi-step immunohistochemical process. The initial step is to bind the primary antibody to its specific epitope. A secondary antibody may be applied to bind with the primary antibody, followed by an enzyme-labelled polymer; or an enzyme-labelled polymer may be applied directly to bind with the primary antibody.

An enzyme-mediate colourimetric reaction proves the detection of the bound primary antibody.

\subsection{Statistical Analysis}

The univariate analysis is done to observe the distribution of each variables using a computer system. Presented in the form of charts and graphs. Statistical analysis of the correlation of each variable in the group experiencing psychological stress and non-stress as compared to the group using the Chi-square test.

\section{Results}

Table 1. General Characteristic

$\begin{array}{lll}\text { Variables } & \mathrm{N}(\%) & \text { Average (Mean } \pm \text { SD) }\end{array}$




\begin{tabular}{lll}
\hline Age (years) & 80 & $38,88 \pm 12,12$ \\
Gender & $32(39,5 \%)$ & \\
$\bullet \quad$ Male & $48(59,3 \%)$ & \\
$\bullet \quad$ Female & $40(50 \%)$ & \\
Psychology & $40(50 \%)$ & \\
$\bullet \quad$ Non stress & & $15,79 \pm 7,65$ \\
- Stress & & $24,03 \pm 12,18$ \\
Morning Cortisol (mean) & & $6,43 \pm 4,53$ \\
$\bullet \quad$ Non-stress & $12,42 \pm 9,25$ \\
- Stress & & \\
Evening Cortisol & $73,28 \pm 16,70$ \\
$\bullet \quad$ Non-stress & $72,95 \pm 19,49$ \\
- Stress & & \\
Average Value IL- 6 & & \\
$\bullet \quad$ Non-stress & \\
- Stress & & \\
\hline
\end{tabular}

From table 1 can be seen the average age of patients $38,88 \pm 12,12$ years, which is women more than men $32(39,5 \%)$ vs $48(59,3 \%)$

Table 2. Serum cortisol level of non-stress and stress groups in the morning and evening.

\begin{tabular}{cll}
\hline Serum Cortisol & (mean) & $\mathrm{P}$ \\
\hline Morning Non-stress & $15,79 \pm 7,65$ & 0,002 \\
Stress & $24,03 \pm 12,18$ & \\
Evening Non-stress & $6,43 \pm 4,53$ & 0,001 \\
Stress & $12,42 \pm 9,25$ & \\
\hline
\end{tabular}

There was a difference in the value of morning cortisol with an evening cortisol value between the two groups and statistically significant. The morning cortisol seen higher in the stress group compared with the non-stress group $(24,03 \pm 12,18$ vs. $15,79 \pm 7,65)$ was statistically significant. The value of evening cortisol also differed between the stress group and the non-stress group $(6,43 \pm 4,53$ vs. $12,42 \pm 9,25)$ and statistically significant.

Table 3. The difference of Interleukin-6 expression in patients suffering from functional dyspepsia with and without psychological stress.

\begin{tabular}{lll}
\hline Group & IL-6 Level (\%) & P \\
\hline Non Stress & $73,28 \pm 16,70$ & 0,428 \\
Stress & $72,95 \pm 19,49$ & \\
\hline
\end{tabular}

There was the difference in the value of IL-6 expression between stress group and non-stress group, but statistically not significant.

Table 4. Analysis of Morning Cortisol serum with IL-6 expression

\begin{tabular}{ccccc}
\hline Group & Cortisol Serum & IL-6 & expression & $\mathrm{P}$ \\
& & & \\
\hline
\end{tabular}




\begin{tabular}{llll}
\hline Non Stress & $15,79 \pm 7,65$ & $73,28 \pm 16,70$ & 0,55 \\
Stress & $24,03 \pm 12,18$ & $72,95 \pm 19,49$ & 0,05 \\
\hline
\end{tabular}

To see the influence of cortisol in the morning to the expression of IL -6 , in table 4 in the non-stress group not significant but in the stress group, it was found significant.

Table 5. Analysis of Evening cortisol serum with IL-6 level

\begin{tabular}{llll}
\hline Group & Cortisol Serum & IL-6 Level (\%) & P \\
\hline Non Stress & $6,43 \pm 4,53$ & $73,28 \pm 16,70$ & 0,18 \\
Stress & $12,42 \pm 9,25$ & $72,95 \pm 19,49$ & 0,06 \\
\hline
\end{tabular}

In table 5, evening cortisol did not influence the expression of IL-6, both of group have no significant result in statistic analysis.

\section{Discussion}

Cortisol hormone released by exposure to psychological stressors will stimulate the production of stomach acid (aggressive factor) and can inhibit prostaglandins (defensive factors) that are protective in the stomach. Reduced prostaglandins for the next will facilitate the occurrence of damage to the gastric mucosa. Andreas's (2005) study proved that there was a significant increase in the value of cortisol in FD compared to the control group. Similarly, Murni study (2006) found a significant increase in morning cortisol in patients with depressed DF $(p=0.019)$. [4][11]. In this study also proved the presence of morning cortisol seen higher in the stress group compared with the non-stress group $(24,03 \pm 12,18$ vs. $15,79 \pm 7,65)$ was statistically significant. The value of evening cortisol also differed between the stress group and the non-stress group $(6,43 \pm 4,53$ vs. $12,42 \pm 9,25)$ and statistically significant

Some inflammatory markers were found to increase in patients with FD, such as TNF a, IL 6, il-12, IFN gamma, IL-1B, IEL / CD3 / CD 25, TLR4, anti-flagellin antibody and there was eosinophil infiltration in the duodenum and elevated esosinophils in fluid Stomach. There is no study determine expression IL-6 based on stress psychological before. This study wants to know the difference of expression IL-6 between stress group and non-stress group. It was found that the expression of IL- 6 was seen less in the psychologically stressful group than in non-stress. It means the expression of IL- 6 could be influenced by other factors such as Helicobacter pylori inflammation or other microbial stimuli that may be more suffered by patients who do not experience psychological stress.

The association of morning cortisol with IL-6 expression also showed a significant association in psychologically stressful groups. In table 4 show that significantly association between morning cortisol with expression IL-6 and not significant in the non-stress group. Activation of the HPA axis may play a role in enhancing the observed inflammatory activity with increased IL-6 expression. This study proves that psychological stress can affect immunity as evidenced by the significant of the relationship of cortisol as a stress hormone with the expression of IL-6. The other study also found that inflammation has an important role in developmental mediator linking adverse experience in early life to poor adult mental health by expression of IL-6. Interleukin-6 was an integral part of the innate immune response to a 
physical stressor such as infection and remain to be determined that psychosocial stressor also initiates cytokine response as well as the clinical consequences of exaggerated cytokine response to stress.[12]

The previous study by Darwin et al. ( 2017) found that percentage of IL-6 in gastric mucous of FD patients with psychological stress were lower than patients without psychological stress. This result is the same with this study (table 3), but analysis relationship expression IL-6 with cortisol level, there is a significant relationship between morning cortisol level and expression IL-6. This condition mentions that psychological stress has an impact of inflammation in gastric mucous, so could be the influence of defensive mechanism of gastric mucous balance. Finally, psychological stress is one of the important elements in the handling of FD and should be a concern in managing the patient to improve clinical symptoms and prevent further mucosal damage.

\section{References}

[1] Mahadeva S, Goh KL. Epidemiology of Functional Dyspepsia: A Global Perspective. World JvGastroenterol.12 (17).pp 2661-2666 (2006)

[2] Cadamuro ACT, Rossi AVT, Maniezzo NM, Silva AE, Helicobacter Pylori Infection: Host Immune response, implication on gene expression and micro RNAs. World J Gastroenterol; 20(6) pp $1424-1437$ (2014)

[3] S,Dinan TG,Barry S 2006. Functional dyspepsia: are psychosocial factors of relevance? World J Gastroenterol; 12(17):2701-07

[4] Dickerson LM, D Pharm, King D. Evaluation and Management of Nonulcer Dyspepsia.Am Fam Physician; 70 : 107-14 (2004)

[5] Darwin E, Murni AWM, Nurdin AE . Affect of Psychological Stress to the Mucosal IL-6 and Helicobacter Pylori Activity in Functional Dyspepsia. Acta Med Indones 2017;49(2); pp 99-104

[6] Bohmelt AH, Nater Urs M, Franke S. Basal and stimulated HPA axis activity in a patient with the functional gastrointestinal disorder and healthy controls. Psychosom Med.2005.67 pp 28894(2005)

[7] Konturek PC, Brzozowski T, Konturek SJ, 2011. Stress and gut: Pathophysiology, clinical consequences, Diagnostic Approach and treatment options; J Physiol Pharmacol; 62(6):591-9

[8] Harris TB, Ferrucci L, Tracy RP, Corti MC, Wacholder S, Ettinger WH, et al.

[9] Association of Elevated interleukin-6 and C-reactive protein levels with mortality in the elderly. Am J Med.; 106 pp 506-12 (1999)

[10] Elhage R, Clemens S, Besnard S, Mallat Z, Tedqui A, Arnal J et all. Involvement of interleukin-6 in atherosclerosis but not in the prevention of fatty streak formation by 17 beta-estradiol in apolipoprotein E-deficient mice. Atherosclerosis.; 156 pp 315-20 (2001)

[11] Jakkimainen R.Liisa, Boyle E, Tudiver F. Is Helicobacter Pylori Associated with Non-Ulcer Dyspepsia and Will Eradication Improve Symptoms? A meta-analysis. BMJ; 319; 1040- 1044 (1999)

[12] Murni AW. Kadar Cortisol Plasma Pada penderita Dyspepsia Fungsional dengan Depresi. Tesis Sp Penyakit Dalam, Padang. Fakultas Kedokteran Universitas Andalas.(2006)

[13] Carpenter LL, Gawuga CE, Tyrka AR, Lee JK, Anderson GM, Price LH. Association between Plasma IL-6 Response to Acute Stress and Early-Life Adversity in Healthy Adults. Neuropsychopharmacol 35 pp2617-23 (2010) 